\title{
Aplicaciones del Programa Leer para Aprender en el nivel universitario: principios, desafíos y adaptaciones
}

\author{
Aplicações do Programa Ler para Aprender no nível universitário: princípios, desafios e adaptações
}

\author{
Margarita Vidal Lizama
}

Pontificia Universidad Católica de Chile - PUC-UC - Santiago - Chile

\begin{abstract}
Resumo: O presente artigo descreve a aplicação e adaptação dos princípios centrais do Programa Ler para Aprender (LPA) para o desenho de um projeto orientado ao desenvolvimento da escrita clínica na área da Fonoaudiologia, numa universidade tradicional chilena. O artigo descreve os princípios centrais de LPA, organizados em dois âmbitos: desenho estratégico e desenho teórico-didático. Revisam-se os desenvolvimentos conceituais mais recentes para ambos os domínios, com foco específico na recontextualização de conceitos próprios da teoria linguística sistêmicofuncional (LSF) na metodologia LPA. Logo, é descrito como estes princípios foram adotados e adaptados para o desenho do projeto em questão. Revisam-se as três etapas do projeto: i) identificação das necessidades de escrita dos estudantes, ii) desenho e aplicação da intervenção baseada em princípios do Programa Ler para Aprender e iii) avaliação do impacto da intervenção. Em cada uma delas, são apresentadas as adaptações realizadas. $\mathrm{O}$ artigo busca oferecer uma alternativa de uso da metodologia LPA em contextos institucionais, nos quais uma aplicação extensa e profunda dela é incompatível com as condições materiais da instituição, e evidenciar os princípios teóricos centrais para obter resultados positivos no processo.
\end{abstract}

Palavras-chave: Ensino da escrita acadêmica, espanhol acadêmico, Ler para Aprender

Resumen: El artículo describe la aplicación y adaptación de los principios centrales del Programa Leer para Aprender (LPA) para el diseño de un proyecto orientado al desarrollo de la escritura clínica en el área de Fonoaudiología en una universidad tradicional chilena. El artículo describe los principios centrales de LPA, organizados en dos ámbitos: diseño estratégico y diseño teórico-didáctico. Se revisan los desarrollos conceptuales más recientes para ambos dominios, con foco específico en la recontextualización de conceptos propios de la teoría lingüística sistémico funcional (LSF) en la metodología LPA. Luego se describe cómo estos principios fueron adoptados y adaptados para el diseño del proyecto en cuestión. Se revisan las tres etapas del proyecto: i) identificación de las necesidades de escritura de los estudiantes, ii) diseño y aplicación de la intervención basada en principios del Programa Leer para Aprender y iii) evaluación del impacto de la intervención. En cada una se presentan las adaptaciones realizadas. El artículo busca ofrecer una alternativa de uso de la metodología LPA en contextos institucionales en los cuales una aplicación extendida y profunda de esta es incompatible con las condiciones materiales de la institución, y hacer evidente los principios teóricos centrales para lograr resultados positivos en el proceso.

Palabras clave: Enseñanza de la escritura académica, español académico, Leer para Aprender. 


\section{Introducción}

El término 'pedagogía de géneros' se utiliza para describir el conjunto de principios y estrategias pedagógicos que han emergido desde la comprensión socio-semiótica del lenguaje planteada por la Lingüística sistémico funcional (en adelante, LSF) (Halliday y Matthiessen, 2004; Martin, 1992; Martin y Rose, 2007; 2008) y la teorización sobre la enseñanza y el aprendizaje desde la sociología de la educación (Bernstein, 1990; 1996) y la sicología social (Vigotsky, 1998). Estos principios dicen relación, en términos generales, con una comprensión del lenguaje como un fenómeno social, vinculado estrechamente con su contexto de uso en la vida social; con una reflexión sobre la manera en que el lenguaje se organiza alrededor de metafunciones para cumplir propósitos sociales en contextos variados; y con una discusión crítica sobre las opciones de significado disponibles a diversos hablantes en una comunidad y la distribución inequitativa de estos recursos semióticos en la estructura social (Martin y Rose, 2007; 2008; Rose y Martin, 2012).

A partir de estos principios teóricos sobre el lenguaje y su posición en la vida social, se han desarrollado de manera progresiva desde la década de los 80' en Australia una serie de propuestas pedagógicas para la enseñanza de la lectura y la escritura. Estas propuestas han evolucionado para responder a las necesidades educativas de grupos de estudiantes que han tenido históricamente un acceso limitado a los recursos semióticos considerados valiosos en la vida social (Rose, 2018a; Rose y Martin, 2012). En su evolución a lo largo del tiempo, las propuestas pedagógicas surgidas en el marco de la pedagogía de géneros se han incorporado de manera progresiva para constituir el Programa Leer para Aprender (en adelante, LPA) (Reading to Learn, en inglés) (Rose, 2018a).

El programa LPA ha sido aplicado en diversos contextos educativos y geográficos, para responder a las necesidades de estudiantes diversos, como en el sistema escolar en Australia y otros países (KartikaNingsih y Rose, 2018a; Humphrey, 2016; Coffin, C.,
Acevedo, C. y Lövstedt, A.C., 2013; 2014; Gouveia, C., 2014), en contextos de educación en inglés como segunda lengua (Harman, 2017; Herazo, 2012; Moore, Schleppegrell y Palincsar, 2018; Moore y Schleppegrell, 2019), en la enseñanza de español como lengua heredada (Colombi, 2009; 2013), y en contextos de educación terciara (Dreyfus, Humphrey, Mahboob y Martin, 2016; Humphrey y Macnaught, 2011; Humphrey y Vidal Lizama, 2020; Moyano, 2007; 2010; 2017; Montes y Vidal Lizama, 2017; Millin, 2011), entre otros. En cada uno de estos contextos, el programa se enfrenta a desafíos y dificultades particulares, que dependen, por una parte, de las necesidades y condiciones materiales y simbólicas de los estudiantes, y por otra, de las condiciones propias de las instituciones y agencias en las que el programa se aplica. Por ejemplo, las condiciones materiales y la participación de docentes y estudiantes serán diferente si la aplicación del programa surge como una necesidad identificada colectivamente, a si la aplicación se determina 'desde arriba', sin consulta ni concierto de los involucrados. Asimismo, el grado de flexibilidad curricular de las prácticas educativas y de compromiso de los directivos y docentes puede determinar también la profundidad con la que el programa consigue ser aplicado.

La propuesta del programa LPA (Rose, 2018a; Rose y Martin, 2012) plantea principios teóricos y pedagógicos claros y sistemáticos que orientan su aplicación. En términos estrictos, el programa supone un plan de formación profesional de los docentes de largo aliento, que involucra además un trabajo profundo en el diseño curricular y evaluativo de los programas, y por lo tanto un alto grado de compromiso de directivos y docentes en una institución educativa. Sin embargo, puede suceder en algunos casos que la institución no considere esta formación como central o que no esté dispuesta a invertir demasiados recursos humanos (y por lo tanto, tiempo) en ella. Frente a estas condiciones, resulta necesario realizar adaptaciones que respondan a los recursos disponibles y que mantengan los principios teóricos y metodológicos principales de la propuesta pedagógica. 
El propósito de este artículo es presentar una experiencia de adaptación de los principios del programa LPA en un contexto de educación universitaria en Chile, en particular vinculada a la enseñanza de la escritura especializada en el ámbito de las ciencias de la salud. La pregunta central que guía este artículo es la siguiente: ¿cómo se puede diseñar y aplicar un programa de enseñanza de la lectura y la escritura académicas que preserve la robustez teórica y didáctica que ofrece el programa LPA y que sea al mismo tiempo flexible a las oportunidades y limitaciones institucionales? Esta pregunta general plantea cuestiones respecto a qué adaptaciones pueden resultar efectivas para mantener el propósito final del programa LPA en su aplicación: reducir la brecha de éxito educativo entre estudiantes de diferentes grupos sociales a un ritmo que permita ofrecer verdaderamente igualdad de oportunidades educativas para todos (Rose 2018a; Martin y Rose, 2008).

El artículo se organiza de la siguiente manera. En primer lugar, se plantean los principios centrales del programa LPA, en relación con su diseño estratégico general, los principios clave sobre el lenguaje que lo sustentan y sus estrategias didácticas. Luego, se describe el proceso de adaptación y aplicación de estos principios en el diseño de una intervención orientada a la enseñanza de la escritura de informes clínicos en el contexto de la carrera de Fonoaudiología. Se discuten aquí las motivaciones de esta intervención, así como las decisiones tomadas y el conocimiento desarrollado en este proceso, incluida la evaluación de su impacto. Finalmente, se discuten brevemente algunos logros y dificultades de este proceso, estableciendo vínculos con otras alternativas de aplicación del programa LPA a la enseñanza de la escritura y lectura académicas desarrolladas en el contexto latinoamericano.

\section{Principios del programa Leer para Aprender: caracterización}

En el programa LPA es posible distinguir dos ámbitos complementarios en los que se proponen sus principios centrales. Estos ámbitos serán llamados aquí i) diseño estratégico y ii) diseño teórico-didáctico. El ámbito del diseño estratégico dice relación con los principios que se proponen para alcanzar una aplicación, integración y consolidación del programa LPA en los contextos educativos que permita su sustentación en el tiempo y su 'naturalización' como la metodología adecuada para acelerar el aprendizaje de todos los estudiantes y reducir la brecha entre distintos grupos. El ámbito del diseño teórico-didáctico se relaciona con los principios conceptuales y teóricos que permiten responder a las preguntas sobre qué enseñar y cómo enseñar desde el marco teóricometodológico ofrecido por la LSF y orientaciones pedagógicas afines a ella. En esta sección se presentarán los principios teóricos relevantes que informan el diseño de LPA, dejando de lado las estrategias específicas de este programa y asumiéndolas como conocidas.

\section{Diseño estratégico}

Respecto de ámbito de diseño estratégico, el programa LPA propone el trabajo sostenido con dos grupos de actores: por una parte, la aplicación de la pedagogía en la sala de clases, con los estudiantes; por otra, el desarrollo profesional de los docentes, quienes deben ser formados en la metodología de enseñanza para aplicarla a sus disciplinas, niveles educativos y propósitos (Rose, 2018a). Ciertamente, la aplicación sostenida en el tiempo de la metodología en la sala de clases requiere que se lleve a cabo una formación profunda y continua de los docentes que llevan a cabo la tarea de enseñanza en un contexto, en vez de confiar en una aplicación fragmentada o no integrada de manera efectiva en el currículo. Para conseguir este objetivo, el entrenamiento progresivo de los docentes resulta fundamental para la consolidación del programa LPA en los contextos educativos en los que se aplique. En el ámbito de la enseñanza de la lectura y la escritura académicas en América Latina, este principio es también planteado por Moyano (2017) como un punto clave para la 
sustentación de programas en el contexto universitario a lo largo del tiempo.

Sin embargo, una distinción importante de la propuesta de Moyano, que adapta la metodología LPA a su contexto de acción, es que, según su modelo, la enseñanza de la lectura y escritura académica debe ser realizada en conjunto por un docente de lengua y un docente de la especialidad disciplinar, y es el docente de lengua quien debe ser entrenado de manera exhaustiva en la metodología LPA. Por su parte, desde la perspectiva de Rose (2018) y su interés en el contexto de educación escolar, la capacitación de los docentes de las diferentes áreas disciplinares relevantes para la escuela es suficiente para alcanzar los propósitos de esta metodología. Esta distinción entre la propuesta original del programa LPA y su aplicación a un contexto para el que no ha sido originalmente diseñada demuestra la necesidad de considerar la naturaleza del contexto de práctica para la aplicación adecuada y orgánica de los principios propuestos por esta metodología.

\section{Diseño teórico-didáctico}

En el ámbito del diseño teórico-didáctico, es posible identificar dos grandes núcleos conceptuales a la base del programa LPA, cada uno de ellos vinculado con las preguntas qué enseñar y cómo enseñar, respectivamente. La primera dice relación con la comprensión del lenguaje en contexto propuesta por la LSF; la segunda, con la concepción de una pedagogía visible y orientada a la generación de cambios en el grupo social (Bernstein, 1996) que se manifiesta a nivel de la organización del currículo, la evaluación del aprendizaje y la interacción en la sala de clases. Estos dos núcleos conceptuales son los que definen de manera más profunda la esencia del programa LPA y lo distinguen de otras metodologías o propuestas didácticas para la enseñanza de la lectura y la escritura.

En el marco del programa LPA, el lenguaje se entiende como un sistema socio-semiótico cuya función principal es la de producir significado y establecer relaciones entre hablantes en contextos sociales. Esta comprensión del lenguaje proviene de la LSF (Halliday y Matthiessen, 2004; Martin, 1992; Martin y Rose, 2007; 2008), y en particular de la propuesta de Martin (Martin, 1992; Martin y Rose, 2007) para modelar la relación entre lenguaje y contexto. En esta propuesta, el contexto es modelado alrededor de dos niveles o estratos, género y registro. El género es entendido en este marco como un proceso social orientado a una meta y dividido en etapas (Martin \& Rose, 2007). Rose (2018a) ofrece una mirada complementaria al describir género como "una configuración regular de campo, tenor y modo, que se despliega en patrones que son predecibles para los miembros de una cultura" (p.63, traducción propia).

Esta descripción del concepto de género hace énfasis en la relación de realización que existe entre género, como el estrato más abstracto del contexto, y registro, como el menos abstracto y el que media entre el contexto y el lenguaje como otro sistema semiótico. El registro se compone de esta manera de tres variables contextuales relevantes: el campo, es decir, la actividad que se está llevando a cabo - y desde el punto de vista del programa LPA, el conocimiento en juego en esta actividad (Rose, 2018a, ver otro); el tenor, es decir, las relaciones que se establecen entre los hablantes en un contexto dado, y el modo, es decir, el rol que cumple el lenguaje en el contexto social y los recursos semióticos mediante los cuales se organiza (oral/auditivo, escrito, visual) (Martin y Rose, 2007; 2008). Género y registro varían de manera independiente: por ejemplo, un tipo de género como el informe puede ser utilizado en ciencias para describir una entidad natural - como una célula - o en arte para describir una entidad cultural - como una escultura (cf. Rose, 2018a).

Las variables de registro y género se realizan en el lenguaje a través de patrones de opciones de significado organizadas en tres tipos - significado ideacional, significado interpersonal y significado textual (también llamadas metafunciones). El significado ideacional dice relación con los recursos para representar personas, cosas y acciones en el lenguaje, así como secuencias de esas acciones; el significado interpersonal refiere a los recursos para 
negociar información (como preguntas y respuestas), así como recursos para elaborar evaluaciones en los textos y establecer posiciones dialógicas de los hablantes. Finalmente, el significado textual se relaciona con los recursos para organizar los textos en 'ondas' informativas que introducen y acumulan información en diferentes puntos (Martin y Rose, 2007; 2008).

Los recursos de significado ideacional, interpersonal y textual se organizan además en el lenguaje en tres niveles o estratos: el estrato semántico-discursivo, que involucra los patrones a lo largo del texto, el estrato léxico-gramatical, vinculado a los recursos propios de la cláusula y el vocabulario; y el estrato fonológico-grafológico, que organiza los sonidos y grafías en una lengua. Entre estos niveles se establece también, tal y como entre el contexto y el lenguaje, una relación de realización: los patrones discursivos en el texto se realizan (codifican, simbolizan) en los patrones gramaticales que a su vez son realizados por los recursos en el estrato fonológico-grafológico. Esta manera de comprender el lenguaje en contexto se representa en la LSF típicamente en una figura de círculos circunvinientes, como en la Figura 1.

Figura 1: modelo de lenguaje en contexto de la LSF (adaptado de Martin y Rose, 2008)

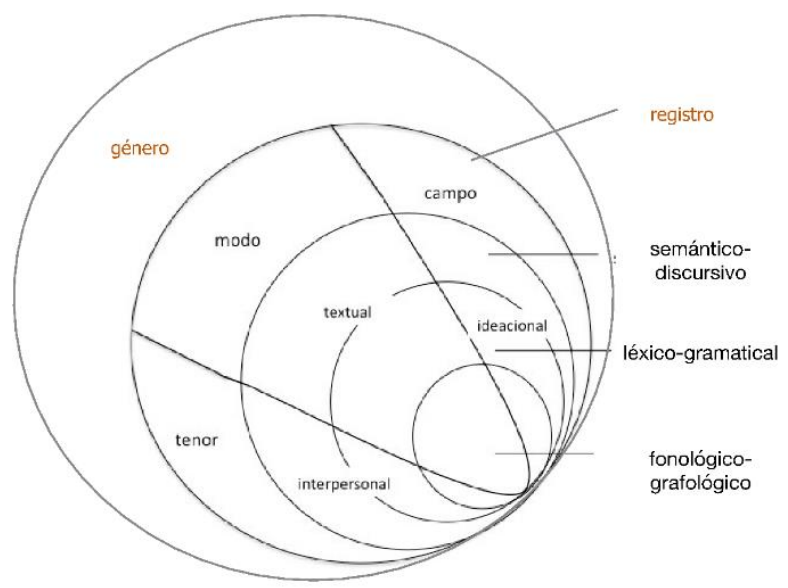

Recontextualizando conceptos de la teoría en el programa LPA
La propuesta de LPA (Rose, 2018a; 2020a; 2020b) integra la recontextualización de conceptos clave relativos a la comprensión del lenguaje desde la LSF para ofrecer una mayor especificidad conceptual en la metodología LPA. Esta recontextualización ha sido un principio relevante en la propuesta pedagógica surgida desde la LSF desde los primeros modelos propuestos (por ejemplo, Christie, 2002). La primera recontextualización importante en LPA dice relación con la noción de género, que se utiliza para distinguir entre géneros de conocimiento (knowledge genres) y géneros curriculares (curriculum genres) (Martin y Rose, 2013; Rose, 2020a; b); la segunda, con la noción de registro y su aplicación para la comprensión de la práctica pedagógica y para la aplicación de estrategias específicas para la enseñanza de la lectura y escritura. A continuación, se describen ambas recontextualizaciones $y$ se introducen algunos desafíos para su aplicación.

Los géneros de conocimiento (Martin y Rose, 2008) corresponden a aquellos tipos de géneros que los estudiantes leen en el contexto educativo para aprender el conocimiento disciplinar relevante $y$ producen para demostrar su aprendizaje. Para el contexto de la escuela primaria y secundaria en Australia, estos géneros de conocimiento han sido descritos y organizados alrededor de tres grandes familias: géneros informativos, géneros evaluativos y géneros para establecer vínculos (engaging genres) (Martin y Rose, 2013).

La identificación y descripción de los géneros de conocimiento propios de la práctica educativa en la que se aplica el programa LPA resulta un punto de partida fundamental para su consolidación exitosa. Este paso es importante, particularmente considerando que la descripción de géneros de conocimiento ofrecida por la LSF emerge inicialmente en contextos de práctica educativa primaria y secundaria en inglés (Martin y Rose, 2008; Humphrey, 2016). En la educación universitaria en el contexto latinoamericano de habla hispana, diversos estudios han abordado la descripción de géneros de conocimiento relevantes para la enseñanza de la lectura y escritura académica desde la perspectiva 
sistémico-funcional (Moyano, 2011; 2015a; 2015b; Navarro y Simões, 2019; Vidal Lizama, 2020, etc.). La descripción de los géneros de conocimiento en el nivel terciario debiera ser un paso inicial en el diseño de una estrategia de enseñanza de lectura y escritura académica (Moyano, 2017; Montes y Vidal Lizama, 2017).

Los géneros curriculares, por su parte, son aquellos géneros mediante los cuales los estudiantes adquieren los géneros de conocimiento relevante en su contexto educativo (Martin y Rose, 2013). El término géneros curriculares fue acuñado por Christie (2002) e incluye las actividades pedagógicas, las relaciones entre docente $y$ estudiante $y$ las diferentes modalidades utilizadas en el discurso de la sala de clases, además del conocimiento (o géneros de conocimiento) proyectados a través de los géneros curriculares. Desde el punto de vista del programa LPA, un género curricular central para los propósitos del programa es el de la micro-interacción en la sala de clases, que se modela en términos de la actividad de aprendizaje (learning activity) (Rose y Martin, 2012; Martin y Rose, 2013).

La actividad de aprendizaje plantea que todo aprendizaje se produce a partir de la realización de una tarea, que es preparada adecuadamente por el docente y usada luego como un trampolín para que este ofrezca conocimiento relevante al estudiante (Rose y Martin, 2012; Rose, 2020b). Una actividad de aprendizaje puede ser leer un texto, responder una pregunta, observar una imagen, identificar una palabra, etc. Esta tarea se utiliza no para evaluar lo que el estudiante sabe (o no sabe), sino para involucrarlo en una interacción en la que el docente entregará conocimiento relevante. La actividad de aprendizaje constituye el género curricular más básico y fundamental en la propuesta del programa LPA, debido a que organiza la interacción entre docente y estudiante para asegurar que esta sea explícita y ofrezca el soporte necesario al estudiante. Un desafío importante respecto de este género curricular se relaciona con los contextos en los que la modalidad pedagógica principal no es la oral, sino la escrita - por ejemplo, en el diseño de materiales de enseñanza cuando no es posible llevar a cabo clases presenciales.

El concepto de registro es también relevante en la propuesta de LPA de Rose (2020a; b). El registro se constituye como un ámbito conceptual central para el diseño de la actividad pedagógica, y en particular, para discutir la necesidad de recontextualizar la teoría lingüística de alta tecnicalidad en un metalenguaje adecuado a la práctica docente (Rose, 2020a), por una parte, y plantear la importancia de la recontextualización de significados en los textos para el abordaje de la lectura en los contextos educativos, por otra (Rose, 2018b; Rose, 2020b). En este proceso de recontextualización, Rose (2020b) plantea la importancia de elaborar un metalenguaje que, informado en la teoría y el conocimiento especializado sobre el lenguaje ofrecido por la LSF, pueda ser aplicado en la práctica pedagógica para la enseñanza explícita de los géneros de conocimiento, sus etapas, sus características registrales (es decir, campo, tenor y modo) y sus patrones discursivos más comunes (en relación con este punto, véase también Humphrey, 2016). Un ejemplo de esta recontextualización es ofrecido por Rose (2020b) y presentado en la Tabla 1.

Tabla 1: recontextualización de la descripción de géneros de conocimiento

\begin{tabular}{|c|c|c|c|}
\hline género & campo & tenor & $\begin{array}{l}\text { patrones } \\
\text { discursivos }\end{array}$ \\
\hline historias & $\begin{array}{l}\text { tramas, } \\
\text { ambiente, } \\
\text { personajes, } \\
\text { temas }\end{array}$ & $\begin{array}{l}\text { placer en la } \\
\text { literatura, } \\
\text { juicios sobre } \\
\text { los personajes }\end{array}$ & $\begin{array}{l}\text { recursos literarios } \\
\text { para atraer a los } \\
\text { lectores y construir } \\
\text { la trama }\end{array}$ \\
\hline $\begin{array}{l}\text { textos } \\
\text { informativos }\end{array}$ & $\begin{array}{l}\text { conocimiento } \\
\text { del mundo } \\
\text { social y } \\
\text { natural }\end{array}$ & $\begin{array}{l}\text { interés en el } \\
\text { conocimiento }\end{array}$ & $\begin{array}{l}\text { estructuración del } \\
\text { conocimiento, } \\
\text { mediante } \\
\text { abstracción y } \\
\text { tecnicalidad }\end{array}$ \\
\hline $\begin{array}{l}\text { argumentos y } \\
\text { respuestas a } \\
\text { textos }\end{array}$ & $\begin{array}{l}\text { problemas, } \\
\text { posiciones, } \\
\text { análisis, } \\
\text { críticas }\end{array}$ & $\begin{array}{l}\text { negotiación de } \\
\text { posiciones } \\
\text { (evaluación } \\
\text { crítica) }\end{array}$ & $\begin{array}{l}\text { estructuración de } \\
\text { argumentos y } \\
\text { evaluación }\end{array}$ \\
\hline
\end{tabular}

Fuente: traducido de Rose (2020b)

El registro es también relevante para el abordaje inicial de los textos en la enseñanza de la lectura, que constituye el primer paso en el set de estrategias propuesto por el programa LPA. Según Rose (2020b), las estrategias Preparación a la lectura y Lectura detallada, con las cuales se inicia la 
metodología, deben focalizarse en primer lugar en el campo, el tenor y el modo de los textos, y recontextualizar en la interacción, mediante la modalidad oral, las características propias del texto en cuestión. Esta recontextualización implica, en palabras simples, decirles a los estudiantes de qué se trata el texto, cuáles son sus temas o significados principales, cómo se organiza, qué hace primero, qué hace luego, etc., antes de llevar a cabo una lectura de este. La enseñanza del género de conocimiento y sus etapas y patrones discursivos solo puede llevarse a cabo una vez que el registro ha sido abordado, 'traducido' a los estudiantes y discutido con ellos. Este foco en el registro es relevante no solo porque asegura que los estudiantes comprendan los textos, sino también porque permite conectar con el conocimiento disciplinar que los docentes tienen sobre sus ámbitos de enseñanza, haciendo relevante para ellos la enseñanza de la lectura como una manera de enseñar el conocimiento de su área.

Esta sección del artículo ha presentado los principios teóricos y metodológicos centrales del programa LPA, tal como han sido elaborados recientemente por Rose (2020a; b). Esta presentación se ha centrado en la vinculación entre la teoría lingüística propuesta por la LSF y la metodología de enseñanza LPA. En la siguiente sección, se describe la aplicación de estos principios a un contexto de educación universitaria en Chile, particularmente en la enseñanza de la escritura especializada en el área de ciencias de la salud.

\section{Aplicando principios de LPA a la enseñanza de escritura especializada en Fonoaudiología: desafíos y decisiones}

\section{Contexto general del proyecto}

La aplicación que aquí se describe se desarrolló como parte de un de desarrollo de la docencia (FONDEDOC) de la Pontificia Universidad Católica de Chile. El objetivo general de este proyecto fue desarrollar e integrar metodologías de enseñanza y evaluación en la docencia disciplinar para el aprendizaje progresivo de la escritura clínica en estudiantes de la carrera de Fonoaudiología, en tres cursos a lo largo del currículo: Introducción a la Fonoaudiología (semestre I), Motricidad Orofacial (semestre IV) y Evaluación de los Trastornos de la Comunicación en niños y adolescentes (semestre VI). Este proyecto fue realizado de manera colaborativa entre una docente de la Escuela de Fonoaudiología, que asumió el rol de Coordinadora del proyecto, y una docente especialista en lingüística educativa, que participó como Colaboradora.

El proyecto fue motivado desde dentro de la Escuela por dos razones particulares. La primera fue la percepción negativa que los docentes disciplinares de diferentes años de la carrera tenían sobre las habilidades de escritura de sus estudiantes en los diferentes cursos del curriculum. Si bien esta percepción sobre la escritura de los estudiantes parece ser extendida en la institución, en el caso de Fonoaudiología se agregaba además la evidencia de sucesivas cohortes en el Examen de Comunicación Escrita (VRA100C) aplicado en la universidad, en el que estos estudiantes obtenían sostenidamente bajos resultados en comparación con el promedio de la institución. Una segunda motivación fue la importancia que los docentes asignan al desarrollo de la escritura, en particular en el contexto de práctica clínica, para el futuro desenvolvimiento profesional de estos estudiantes en equipos médicos constituidos por diferentes profesionales de la salud. Desde el punto de vista de la LSF, la situación de los estudiantes de Fonoaudiología parecía reflejar, por una parte, un acceso limitado a los recursos semióticos necesarios para el éxito en la práctica académica, tal como indicaba su desempeño en los cursos y en el Examen de Comunicación Escrita. Por otra, las expectativas curriculares y de la práctica profesional hacían evidente la importancia de desarrollar esos recursos semióticos para su desempeño tanto dentro como fuera del ámbito universitario. (cf. Martin y Rose, 2008, pp.226-229).

Las condiciones materiales y culturales de la institución en general y del proyecto en particular supusieron constricciones clave para su desarrollo. Al inicio del proyecto, la expectativa de los docentes de 
Fonoaudiología ${ }^{1}$ era contar con materiales de apoyo que los estudiantes pudieran utilizar de manera independiente y fuera de las actividades regulares de enseñanza. Sin embargo, la docente especialista en lingüística educativa planteó la importancia de que los docentes tomaran responsabilidad por la enseñanza de la escritura clínica, para lo que su formación era central. Además, la mayoría de los docentes en la Escuela de Fonoaudiología eran docentes-hora, lo que dificultaba su disponibilidad horaria y afectaba su nivel de compromiso con el proyecto. Finalmente, tanto la Escuela como la Vicerrectoría Académica, patrocinante del proyecto, solicitaban que el proyecto pudiera presentar resultados evidentes y sostenibles en el mediano y largo plazo, y que todo esto fuera realizado durante el año académico de duración del proyecto.

Las condiciones descritas se diferencian claramente de las condiciones planteadas por Rose (2020b), particularmente para el desarrollo progresivo de la formación de docentes en la metodología LPA. En el marco del programa LPA, la formación profesional de los docentes se lleva a cabo mediante un ciclo que incluye una fase de taller/enseñanza cara a cara, seguido de una fase de práctica para terminar con una fase de reflexión conjunta, el que se itera a lo largo de un periodo de tiempo (Rose, 2020b). Este ciclo involucra además la enseñanza progresiva del conocimiento especializado sobre el lenguaje y el metalenguaje relevante para enseñar lectura y escritura. Esta formación profesional de los docentes es posible en ciertas condiciones materiales y culturales, que no eran las del proyecto desarrollado en Fonoaudiología. Esto implicó una serie de decisiones y adaptaciones en el diseño estratégico de LPA, que intentaron preservar los principios centrales de su diseño teórico-didáctico. Estas adaptaciones son detalladas a continuación, indicando su aplicación en cada etapa del proyecto.

${ }^{1}$ En el proyecto participaron un total de 11 docentes, incluyendo la Coordinadora del proyecto. Estos docentes correspondieron a los equipos de Introducción a la Fonoaudiología y Evaluación de los trastornos de la comunicación de niños y adolescentes. Los docentes del equipo de Motricidad orofacial tuvieron una participación limitada, ya que no se logró involucrarlos en la etapa
Aplicación de los principios teóricos de LPA para la enseñanza en Fonoaudiología

El proyecto se organizó en tres etapas: i) levantamiento de información; ii) diseño y aplicación de la intervención y iii) evaluación de la intervención. En la primera etapa, el objetivo central fue identificar los géneros de conocimiento relevantes en la enseñanza progresiva de la práctica clínica de los estudiantes de Fonoaudiología; en la segunda, el foco estuvo en diseñar y aplicar talleres de formación con docentes y actividades de enseñanza en el aula con los estudiantes, así como también la elaboración de materiales de enseñanza/aprendizaje para ambos grupos; finalmente, la tercera etapa evaluó cualitativa y cuantitativamente el impacto de las estrategias aplicadas. En cada una de estas etapas se pusieron en juego diferentes principios teóricos subyacentes en el programa LPA, como se detallará a continuación.

\section{Etapa 1: Levantamiento de información: géneros de conocimiento y concepciones de los docentes}

La primera etapa del proyecto tuvo dos objetivos centrales: por una parte, identificar y caracterizar los géneros de conocimiento que los estudiantes de Fonoaudiología deben conocer para especializarse progresivamente en la práctica clínica en su área; por otra, identificar las concepciones que los docentes de Fonoaudiología tenían sobre la escritura en el campo clínico, sobre las habilidades de escritura de sus estudiantes, y sobre su propio rol en la enseñanza de estas habilidades. Esta información fue de utilidad para elaborar más tarde un diseño que pudiera apelar a las concepciones de los docentes, al mismo tiempo que les entregaba las herramientas necesarias para enseñar de manera explícita y visible la escritura especializada en su área. Este punto era muy importante, debido al tiempo acotado de aplicación del proyecto y la consecuente limitación de la participación de los docentes en talleres de

de formación docente. Sin embargo, igualmente se diseñaron materiales para el curso y se llevaron a cabo dos sesiones con los estudiantes, a cargo del equipo de lingüistas del proyecto. 
formación progresivos que pudieran abordar y modificar sus representaciones respecto de la escritura, el lenguaje y su propio rol en su enseñanza. La etapa de levantamiento de información se llevó a cabo durante el primer semestre del año académico en que se desarrolló el proyecto.

Para llevar a cabo la identificación de los géneros de conocimiento, se llevó a cabo un análisis de género, a partir de la metodología planteada por Hood (2010). Se recolectaron para ello los textos escritos por los estudiantes en el marco de cada curso como parte de las evaluaciones, seleccionando aquellos con más altas calificaciones para el análisis y un grupo con bajas calificaciones como grupo control. El análisis implicó la identificación de patrones textuales, interpersonales e ideacionales instanciados en los textos en el estrato semántico-discursivo. En particular, se realizaron análisis de PERIODICIDAD, VALORACIÓN, IDEACIÓN y CONJUNCIÓN (Martin y Rose, 2007; 2008). A partir de este análisis, se identificaron dos géneros de conocimiento relevantes en la enseñanza de la práctica clínica en Fonoaudiología: el informe de visita y el informe fonoaudiológico.

Para propósitos de la aplicación pedagógica de este conocimiento, se llevó a cabo una recontextualización de la descripción de estos géneros, siguiendo el ejemplo propuesto por Rose (2020b). De esta forma, los dos géneros de conocimiento identificados fueron presentados a docentes y estudiantes considerando un metalenguaje que buscaba representar de manera accesible sus características centrales en términos de registro y patrones discursivos. Esta recontextualización se presenta en la Tabla 2.
Tabla 2: recontextualización de los géneros de conocimiento en Fonoaudiología

\begin{tabular}{|c|c|c|c|}
\hline género & campo & tenor & $\begin{array}{l}\text { patrones } \\
\text { discursivos }\end{array}$ \\
\hline $\begin{array}{l}\text { informe de } \\
\text { visita }\end{array}$ & $\begin{array}{l}\text { observaciones } \\
\text { de la práctica } \\
\text { fonoaudiológica, } \\
\text { espacio de } \\
\text { trabajo, } \\
\text { pacientes, } \\
\text { procedimientos }\end{array}$ & $\begin{array}{l}\text { evaluación de } \\
\text { la importancia } \\
\text { del rol del } \\
\text { fonoaudiólog } \\
\text { o, } \\
\text { identificación } \\
\text { de desafíos }\end{array}$ & $\begin{array}{l}\text { descripción } \\
\text { 'objetiva' de } \\
\text { aspectos } \\
\text { observados; uso } \\
\text { de secuencia en } \\
\text { procedimientos; } \\
\text { recursos de } \\
\text { evaluación }\end{array}$ \\
\hline $\begin{array}{l}\text { informe } \\
\text { fonoaudiológico }\end{array}$ & $\begin{array}{l}\text { conocimiento } \\
\text { técnico de las } \\
\text { funciones del } \\
\text { lenguaje y } \\
\text { estructuras } \\
\text { productivas/rece } \\
\text { ptivas; estado } \\
\text { general del } \\
\text { paciente }\end{array}$ & $\begin{array}{l}\text { evaluación de } \\
\text { grados de } \\
\text { normalidad/a } \\
\text { decuación de } \\
\text { funciones y } \\
\text { estructuras; } \\
\text { negociación } \\
\text { de posibles } \\
\text { acciones }\end{array}$ & $\begin{array}{l}\text { estructuración } \\
\text { del } \\
\text { conocimiento, } \\
\text { mediante } \\
\text { tecnicalidad; } \\
\text { taxonomías de } \\
\text { composición } \\
\text { (parte-todo); } \\
\text { recursos para } \\
\text { evaluar y } \\
\text { graduar }\end{array}$ \\
\hline
\end{tabular}

La recontextualización de la descripción de los géneros de conocimiento en estos términos permitió negociar con los docentes aspectos que para ellos resultaba relevantes en la escritura de cada uno de estos géneros, y orientar la discusión hacia el conocimiento especializado que se esperaba que los estudiantes demostraran en la escritura de los dos tipos de informes. En este sentido, por ejemplo, la descripción del tenor en términos de una 'evaluación de la importancia del rol del fonoaudiólogo' en el informe de visita resultó coherente con las expectativas previas de los docentes respecto de una de las etapas de este género y permitió explicitar esta expectativa a los estudiantes; del mismo modo, la referencia a una 'descripción 'objetiva' de los aspectos observados' como parte de los patrones discursivos constituyó un punto de entrada accesible para discutir con los docentes y estudiantes cómo se realiza en los textos el efecto de objetividad - en otras palabras, reflexionar sobre los recursos de significado ideacional e interpersonal de un modo que conectara con el conocimiento de docentes y estudiantes sobre el campo en juego.

\section{Etapa 2: Diseño y aplicación de la intervención}

Una vez que se identificaron los géneros de conocimiento y se abordaron las creencias de los docentes sobre la escritura clínica en su práctica, el 
proyecto se centró en diseñar las diferentes actividades pedagógicas que integraron la intervención. El objetivo fue crear una secuencia de actividades lo más eficiente y útil posible, tanto para los docentes como para los estudiantes de Fonoaudiología, y que permitiera lograr resultados visibles a partir de una aplicación relativamente breve (un semestre académico). Esta secuencia consideró en primer lugar las fechas de entrega de las dos evaluaciones escritas de los cursos en los que se trabajó la escritura clínica. La secuencia de trabajo se presenta en la Figura 2.

\section{Figura 2: secuencia de trabajo del proyecto}

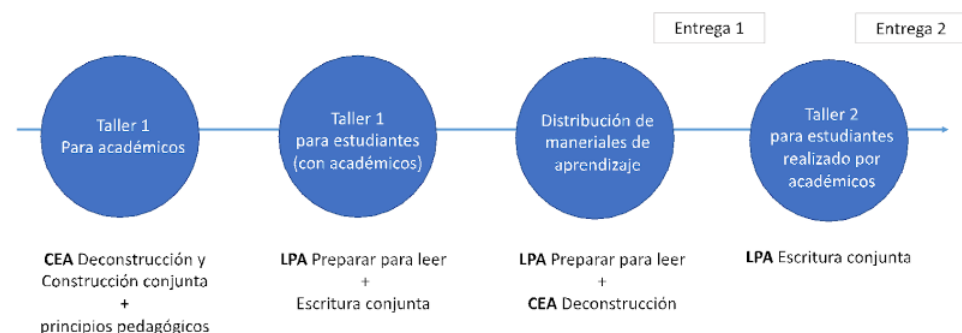

La secuencia comenzó con talleres para los académicos a cargo de los cursos de práctica clínica en diferentes puntos del currículo de Fonoaudiología. El objetivo de estos talleres fue discutir con los docentes los principios pedagógicos centrales de LPA, particularmente respecto de la importancia de una pedagogía visible, de la relación entre conocimiento y lenguaje y de su rol como docentes en la distribución entre todos los estudiantes de los recursos semióticos valorados en su práctica profesional. Esta discusión fue guiada por los resultados obtenidos en los focus groups en la primera etapa del proyecto, de modo que durante el taller se pudo abordar, aclarar y discutir cuestiones clave, como qué aspectos del lenguaje son relevantes de enseñar y cuáles son secundarios (como la ortografía y otros aspectos formales, por ejemplo), cómo es posible integrar la enseñanza de estos en el currículo y el nivel de conocimiento implícito que los docentes tienen de la escritura adecuada en su propio contexto de práctica docente y profesional.

Junto con lo anterior, el taller presentó y modeló las estrategias de Deconstrucción y Construcción conjunta del Ciclo de EnseñanzaAprendizaje (CEA) (Rothery, 1994) con los docentes.
Estas se ofrecieron como una alternativa para desarrollar la enseñanza de la escritura en el área, en particular como parte de una preparación más explícita para la evaluación escrita en los diferentes cursos. La aplicación de estas estrategias con los docentes hizo visible para ellos aspectos interesantes, como sus expectativas sobre la escritura de los estudiantes, la claridad de las instrucciones entregadas para la tarea, el conocimiento que los estudiantes tienen del campo para elaborar cada evaluación y la complejidad de escribir textos adecuados sin los recursos semióticos necesarios.

El segundo paso de la secuencia fue un taller dirigido a los estudiantes de los diferentes cursos involucrados en el proyecto y llevado a cabo por la docente especialista en lingüística educativa con la presencia del docente de Fonoaudiología. Estos talleres se realizaron en diferentes semanas del semestre, pero todos antes de una entrega de evaluación escrita por parte de los estudiantes. Los talleres aplicaron las estrategias Preparar para leer y Escritura conjunta orientadas a la escritura del informe de visita y el informe fonoaudiológico según correspondiera en cada curso. El objetivo de los talleres fue hacer consciente a los estudiantes de las expectativas de la tarea, así como también hacer visible con ellos aspectos de la estructura de los géneros y de sus patrones discursivos. Para estos talleres se entregó a los estudiantes copias de evaluaciones de años anteriores, las que fueron preparadas y leídas en conjunto, para luego practicar brevemente la escritura conjunta de una de las etapas de cada género. Los estudiantes tuvieron la oportunidad de plantear preguntas específicas sobre la evaluación y discutir aspectos del campo con el docente de Fonoaudiología.

El tercer paso de la secuencia fue el desarrollo y distribución de materiales de aprendizaje para estudiantes y materiales de apoyo y evaluación para docentes. Los materiales para los estudiantes aplicaron las estrategias de Preparar para leer y Deconstrucción en recursos escritos y audiovisuales (mini-videos de entre 2-3 minutos), para consolidar lo aprendido durante el taller presencial. Estos materiales 
se desarrollaron también como una manera de asegurar que, una vez terminado el proyecto, los estudiantes de subsiguientes cohortes tendrían algún tipo de apoyo para el desarrollo de la escritura en su área, incluso si los docentes decidían no enseñarla de manera explícita en la clase. Tal y como plantea Rose (2020b), el foco de Preparar para leer estuvo puesto en recontextualizar aspectos del registro, mientras que la Deconstrucción se centró en la estructura de los géneros y sus principales patrones discursivos. Ejemplos de fragmentos de los materiales escritos se presentan en la Figura 3.

\section{Figura 3: ejemplos de materiales escritos para estudiantes}

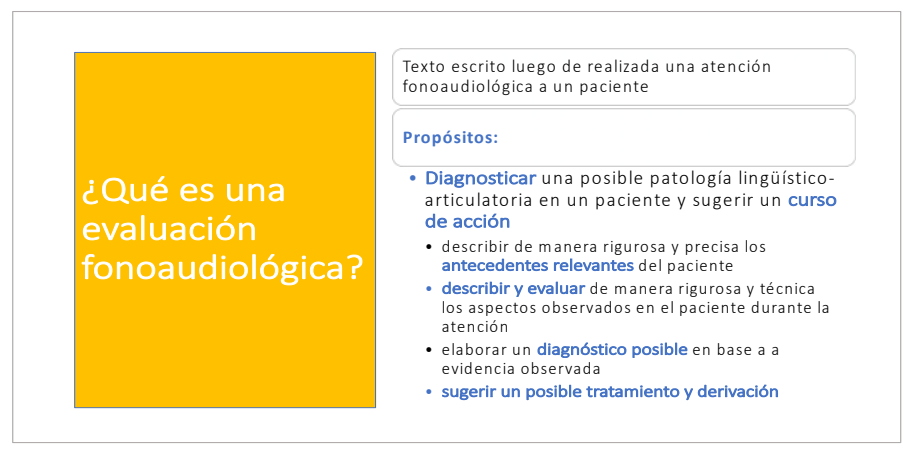

Estructura general de la evaluación fonoaudiológica

\begin{tabular}{|c|c|}
\hline Etapa & Descripción \\
\hline Antecedentes del paciente & $\begin{array}{l}\text { En esta etapa se describen: } \\
-\quad \text { Los datos personales del paciente (nombre, edad, etc.) } \\
\text { - El motivo de consulta } \\
\text { - Los antecedentes relevantes (generales y mórbidos) }\end{array}$ \\
\hline Evaluación & $\begin{array}{l}\text { En esta etapa se describen: } \\
\text { - Los instrumentos utilizados en la evaluación } \\
\text { - La actitud personal del paciente durante la examinación } \\
\text { - Los aspectos observados durante el diagnóstico } \\
\text { - Una síntesis general de los aspectos observados }\end{array}$ \\
\hline Diagnóstico & $\begin{array}{l}\text { En esta etapa, se propone un diagnóstico en base a la } \\
\text { examinación realizada y a los antecedentes descritos } \\
\text { previamente }\end{array}$ \\
\hline Curso de acción & $\begin{array}{l}\text { En esta etapa, se proponen: } \\
\text { - Recomendaciones al paciente, a partir de los } \\
\text { antecedentes descritos y la diagnosis realizada } \\
\text { - Posibles derivaciones a otros especialistas de salud }\end{array}$ \\
\hline
\end{tabular}

Para los docentes, se desarrolló un manual de escritura académica para Fonoaudiología, en el que se presentaron en modalidad escrita los principios pedagógicos y lingüísticos discutidos en los talleres, se describieron los géneros de manera detallada y se ofrecieron alternativas de estrategias pedagógicas para aplicar la enseñanza en la sala de clases o mediante otras actividades. Junto con esto, se elaboraron rúbricas de evaluación de las tareas de escritura, adaptando los principios planteados en Dreyfus et al (2016) para la evaluación. La principal adaptación fue la organización de la rúbrica en etapas del género en vez de niveles de lenguaje, como presentan las autoras. Esto se debió a dos razones: para los docentes, la estructura genérica resulto más comprensible y accesible que las distinciones entre recursos de niveles de lenguaje; y los docentes necesitaban evaluar con distinta ponderación algunas de las etapas del género, debido a su complejidad disciplinar. Para cada una de las etapas descritas se mantuvo el registro como foco central para la descripción del nivel de logro, y en particular los aspectos relevantes del campo en cada una de las etapas. Estas rúbricas fueron aplicadas por todos los docentes en cada uno de sus cursos, lo que permitió también unificar la evaluación y por lo tanto potenciar la enseñanza de los aspectos evaluados.

La secuencia involucró como último paso la aplicación de un taller por parte de los docentes de Fonoaudiología, previo a la entrega de la segunda evaluación del curso. Estos talleres se realizaron en tres secciones del curso Introducción a la Fonoaudiología y en una sección del curso Evaluación de los trastornos de la comunicación. Los talleres fueron videograbados para contar con datos para la evaluación del impacto, pero debido a restricciones de tiempo estos no pudieron ser analizados.

La secuencia presentada tuvo como propósito integrar, en el periodo de un semestre académico (cuatro meses calendario), la aplicación del programa LPA con los estudiantes y la formación de los docentes de cada curso en esta metodología. El diseño de esta secuencia buscó introducir progresivamente a los docentes a los principios centrales de LPA, ya fuera en talleres dirigidos directamente a ellos, en la posibilidad de observar y participar de la aplicación de la metodología realizada por la docente-especialista en lingüística educativa con los estudiantes, en el acceso a materiales escritos y en la práctica de las estrategias en su propia sala de clases. En este sentido, la secuencia intentó replicar la progresión planteada por LPA para la formación profesional de los docentes, 
teniendo en cuenta las limitaciones del contexto en cuestión.

\section{Etapa III: evaluación de la intervención}

La última etapa de la aplicación del proyecto fue la evaluación de su impacto. En el programa LPA este es un punto crucial para demostrar la efectividad de la metodología para la enseñanza de la lectura y la escritura. En aplicaciones de LPA en el contexto escolar, una serie de reportes han demostrado su efectividad, a partir de la recolección de datos cualitativos (encuestas y entrevistas de autopercepción) y datos cuantitativos (resultados en tareas de lectura y escritura pre y post-intervención) (Coffin, Acevedo, y Lövstedt, 2013; 2014; Gouveia, 2014). Para el proyecto se adaptaron estos principios de evaluación, debido a que no se diseñaron tareas preintervención específicas para observar el impacto en los resultados de escritura, sino que se consideraron evaluaciones de cohortes anteriores como punto de partida para la comparación. Para establecer una comparación adecuada, un evaluador aplicó las rúbricas elaboradas en el proyecto para volver a evaluar los informes de la cohorte anterior, de modo que ambas cohortes fueron medidas de igual manera. Junto con esto, se aplicaron encuestas de autopercepción y satisfacción a docentes y a estudiantes, en vez de entrevistas de auto-percepción solamente a docentes. Esta adaptación buscaba identificar no solo la perspectiva de los docentes en el proceso, sino también las percepciones de los estudiantes sobre la utilidad de las actividades realizadas para su propio aprendizaje.

Los datos recolectados para la evaluación del impacto señalaron que, en términos generales, la intervención fue evaluada positivamente tanto por docentes como por estudiantes. De un total de 197 estudiantes involucrado en el proyecto, un $74 \%$ respondió la encuesta de evaluación. De este grupo, un $80,2 \%$ evaluó los talleres de escritura como 'muy útil' y 'útil'. En el grupo docente, se pudo aplicar la encuesta a 9 de los 11 participantes, debido a que dos de ellos no se encontraban trabajando en la Escuela al momento de la aplicación. De este grupo, 8 señalaron estar satisfechos de manera global con los talleres realizados y evalúan positivamente el diseño de materiales de enseñanza-aprendizaje. La evaluación positiva de los docentes se relacionó tanto con un cambio en la concepción de la escritura y el lenguaje en la disciplina, como con la metodología aplicada para la intervención.

Respecto del impacto en el desempeño de los estudiantes en las tareas de escritura, la progresión de los estudiantes involucrados en la intervención mostró una mejora significativa a lo largo del tiempo, desde la primera a la tercera entrega de sus evaluaciones, así como una mejora en comparación con la progresión de estudiantes de la cohorte anterior. Estos resultados se presentan en el Gráfico 1.

\section{Gráfico 1: progresión en las calificaciones de cohortes con y sin intervención}

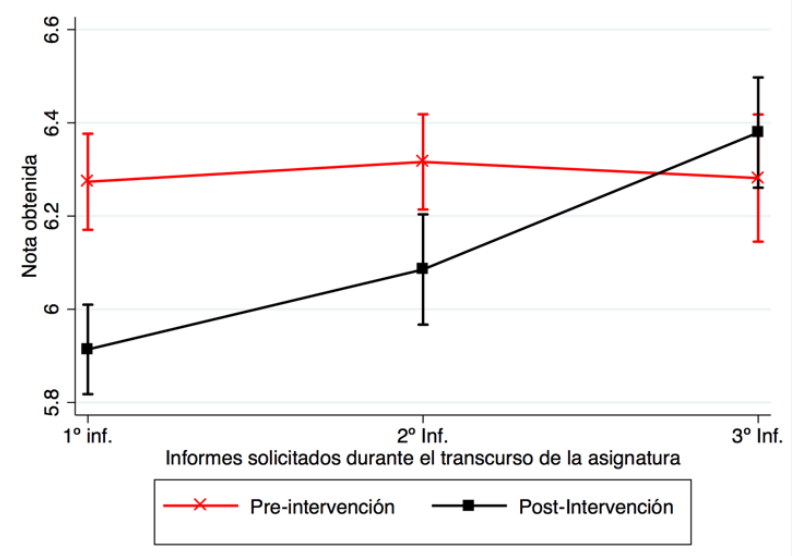

Tal como se observa en el gráfico, la cohorte anterior (en rojo, sin intervención) comenzó con mejores resultados que la cohorte intervenida, pero no tuvo una progresión durante el semestre en términos de las evaluaciones obtenidas. En cambio, la cohorte intervenida tuvo una clara mejora en sus evaluaciones a lo largo del tiempo, incluso habiendo comenzado en un punto de desempeño más bajo. Estos datos reflejan que la aplicación de la metodología LPA, incluso con las adaptaciones llevadas a cabo, tuvo un impacto positivo en la disminución de la brecha de conocimiento de los estudiantes. 


\section{Conclusión}

La metodología LPA ha sido desarrollada a lo largo de décadas de aplicación y teorización en la escuela primaria y secundaria en Australia, subsumiendo en ella sucesivas propuestas pedagógicas basadas en el género y la comprensión socio-semiótica del lenguaje (cf. Rose, 2013; 2018a). Su utilización se ha extendido a diferentes contextos nacionales y educativos en el mundo en los últimos años. Más allá de las diferencias potenciales en las diferentes aplicaciones, todas ellas han tenido como objetivo reducir la brecha de éxito entre estudiantes con diferente capital semiótico y potenciar así la equidad en la sala de clases.

En el contexto de la educación universitaria en español en Latinoamérica, la metodología LPA ha sido integrada de manera exitosa para el diseño y aplicación de programas de lectura y escritura académica, particularmente en el contexto argentino (Moyano, 2010; 2017). En esta aplicación, las adaptaciones han abordado tanto el diseño estratégico como el diseño teórico-didáctico. Respecto del primero, Moyano (2017) ha propuesto la 'negociación', la 'transversalidad' y el 'seguimiento' como estrategias relevantes para la consolidación de un programa basado en LPA en el contexto local. Respecto del segundo, la autora ha adaptado el Ciclo de Enseñanza-Aprendizaje para integrar una etapa de Edición conjunta, teniendo en cuenta las necesidades y el apoyo requerido por los estudiantes para lograr los objetivos de su programa. Estas experiencias dejan de manifiesto la importancia de trabajar con la metodología LPA de manera flexible, adaptando aquellos aspectos necesarios pero manteniendo al mismo tiempo los principios conceptuales clave que la caracterizan y la hacen exitosa.

Este artículo ha presentado una alternativa de aplicación de la metodología LPA en un contexto académico en el que las condiciones no permiten una intervención de alto alcance, como las desarrolladas por Moyano $(2010 ; 2017)$. La aplicación presentada tuvo como pilar fundamental mantener los principios teórico-didácticos de la metodología LPA, en particular aquellos relativos a la comprensión socio-semiótica del lenguaje, al foco en el registro como un punto de entrada para la enseñanza de la escritura disciplinar, al principio de recontextualización en la práctica de enseñanza y a la aplicación de una pedagogía visible en las actividades pedagógicas. El proyecto aquí presentado asumió estos como los principios basales clave para respetar la naturaleza epistemológica y ética del programa LPA.

Uno de los desafíos más importantes de esta aplicación fue el aseguramiento de su permanencia a través del tiempo. En el desarrollo de la intervención quedó de manifiesto la importancia de la formación progresiva de los docentes en la metodología LPA (cf. Rose, 2018a; 2020b) así como la necesidad de aplicar la estrategia de negociación (Moyano, 2017) para lograr un mayor involucramiento y compromiso de actores institucionales clave. Sin embargo, el contexto de realización del proyecto, que supuso tiempo y recursos humanos limitados, y las prácticas y concepciones sobre la escritura, su enseñanza y su relación con el aprendizaje disciplinar, propias de los miembros de la comunidad de Fonoaudiología, no permitieron poner en práctica de manera cabal los principios que fundamentan el programa LPA. Debido a esto, el proyecto buscó proponer adaptaciones que pudieran ofrecer un apoyo visible a lo largo del tiempo para que los docentes de Fonoaudiología pudieran enseñar la escritura clínica y para que los estudiantes pudieran ampliar sus recursos semióticos para esta tarea. Estas adaptaciones involucraron el diseño de géneros curriculares (materiales de enseñanza para estudiantes y materiales de apoyo en la escritura disciplinar en el área para docentes) y complementaciones metodológicas para la descripción de los géneros (aplicación de focus groups a docentes) y para la evaluación del impacto de la intervención (encuestas a docentes y estudiantes). La efectividad de estas adaptaciones para asegurar la permanencia de los resultados positivos alcanzados más allá del periodo de aplicación del proyecto es un aspecto que requiere de un estudio detallado.

A pesar de estos desafíos, la aplicación y adaptación de la metodología LPA en el contexto de 
Fonoaudiología demostró tener un impacto positivo en el desempeño de los estudiantes en la escritura clínica. Esto ciertamente platea la pregunta de cuánto más podrían estos estudiantes crecer en su potencial semiótico para usar el lenguaje en su contexto de aprendizaje - y en su vida social - si pudiera aplicarse la metodología de manera total. Para esto, resulta fundamental poder aplicar las estrategias necesarias planteadas por Moyano (2017) para la consolidación de programas de enseñanza de lectura y escritura académicas en el contexto universitario.

Por otro lado, la aplicación de la metodología LPA, y en particular la discusión de sus principios teórico-pedagógicos en talleres con docentes permitió hacerles conscientes del rol del lenguaje en la enseñanza y el aprendizaje de conocimiento especializado en su área, de la necesidad de enseñar la lectura y la escritura de manera visible en su práctica, y de la importancia de promover una pedagogía que democratice los recursos semióticos. Es de esperar que esta consciencia pueda abrir en el futuro cercano un camino para una aplicación de la metodología LPA en el contexto universitario chileno, que permita alcanzar los claros resultados que este programa ha demostrado tener en otros contextos de aplicación para disminuir la brecha de conocimiento y éxito académico y acercarse así a una estructura social más equitativa.

${ }^{1}$ En el proyecto participaron un total de 11 docentes, incluyendo la Coordinadora del proyecto. Estos docentes correspondieron a los equipos de Introducción a la Fonoaudiología y Evaluación de los trastornos de la comunicación de niños y adolescentes. Los docentes del equipo de Motricidad orofacial tuvieron una participación limitada, ya que no se logró involucrarlos en la etapa de formación docente. Sin embargo, igualmente se diseñaron materiales para el curso y se llevaron a cabo dos sesiones con los estudiantes, a cargo del equipo de lingüistas del proyecto.

\section{REFERÊNCIAS}

BERNSTEIN, Basil. Class, codes and control (Vol. IV The Structuring of Pedagogic Discourse). London: Routledge, 1990.

BERNSTEIN, Basil. Pedagogy, symbolic control and identity. London: Taylor and Francis, 1996.

CHRISTIE, Francis. Classroom Discourse Analysis. London: Continuum, 2002.
COFFIN, Caroline; ACEVEDO, Claire; LÖVSTEDT, AC. Final Report, Teacher Learning for European Literacy Education (TeL4ELE). The Hague: European Union, 2013.

COFFIN, Caroline; ACEVEDO, Claire; LÖVSTEDT, AC. Sweden Project Outputs, Teacher Learning for European Literacy Education. Stockholm Education Administration, The Hague: European Union, 2014.

COLOMBI, Cecilia. A systemic functional approach to teaching Spanish for heritage speakers in the United States. Linguistics and Education, v 20, n 1, p. 39-49, 2009.

COLOMBI, Cecilia. Alfabetización avanzada y exploraciones lingüísticas de los niveles avanzados del español como lengua heredada en los Estados Unidos. Lenguas en contexto, año 10, n 10, p. 54-63, 2013.

DREYFUS, Shoshana et al. Genre Pedagogy in Higher Education. The SLATE Project. London: Palgrave McMillan, 2016.

GOUVEIA, Carlos. Portugal Project Outputs, Teacher Learning for European Literacy Education. Lisbon: Instituto de Linguística Teórica e Computacional (ILTEC), The Hague: European Union, 2014.

HALLIDAY, M. A. K.; MATTHIESSEN, M. I. M. An Introduction to Functional Grammar. $2^{\mathrm{a}}$ edición. London: Oxford University Press, 2004.

HARMAN. M.R. (Ed). Bilingual Learners and Social Equity: Critical Approaches to Systemic Functional Linguistics. Springer: Georgia, USA, 2017, 262 p.

HERAZO, José. Using a genre-based approach to promote oral communication in the colombian english classroom. Colombian Applied Linguistics Journal, v 14, p.109-126, 2012.

HOOD, Susan. Appraising Research: Evaluation in Academic Writing. London: Palgrave Macmillan, 2010.

HUMPHREY, Sally; MACNAUGHT, Lucy. Revisiting Joint Construction in the tertiary context. Australian Journal of Language and Literacy, n 34, p. 98-114, 2011.

HUMPHREY, Sally. Academic Literacies in the Middle Years: A Framework for Enhancing Teacher Knowledge and Student Achievement. London: Routledge, 2016.

KARTIKA-NINGSIH, Harni; ROSE, David. Language shift: analysing language use in multilingual classroom interactions. Functional Linguistics, v 5, p.1-22, 2018.

MARTIN, J. R. English Text. System and Structure. Philadelphia: John Benjamins, 1992. 
MARTIN, J. R.; ROSE, David. Working with discourse. Meaning beyond the clause. London: Continuum, 2007.

MARTIN, J. R.; ROSE, David. Genre Relations. Mapping Culture. London: Equinox, 2008.

MARTIN, J. R., ROSE, David. Pedagogic Discourse: Contexts of Schooling. RASK: International Journal of Language and Communication, n 38, p.219-264, 2013.

MILLIN, T. Scaffolding Academic Literacy with Undergraduate Social Science Students at the University of KwaZulu-Natal using the Reading to Learn Intervention Strategy: An Evaluative Study. MSc Dissertation, The University of Edinburgh Moray House School of Education, 2011.

MONTES, Soledad; VIDAL LIZAMA, Margarita. Diseño de un programa de escritura a través del curriculum: opciones teóricas y acciones estratégicas. Lenguas Modernas, n 50, p. 73-90, 2017.

MOORE, Jason; SCHLEPPEGRELL, Mary; PALINCSAR, Annemarie. Discovering Disciplinary Linguistic Knowledge With English Learners and Their Teachers: Applying Systemic Functional Linguistics Concepts Through Design-Based Research. TESOL Quarterly, v 52, n 4, p. 1022-1049, 2018.

MOORE, Jason; SCHLEPPEGRELL, Mary. A focus on disciplinary language: Bringing critical perspectives to reading and writing in science. Theory Into Practice, $v$ 59, n 1, p. 99-108, 2019.

MOYANO, Estela Inés. Enseñanza de Habilidades Discursivas en Español en Contexto Pre-universitario: Una Aproximación desde la LSF. Revista Signos, v 40, n 65, p. 573- 608, 2007.

MOYANO, Estela Inés. Escritura académica a lo largo de la carrera: un programa institucional. Revista Signos, v 43, n 74, p.465-488, 2010.

MOYANO, Estela Inés. La Discusión de los artículos de investigación en el área de la agronomía: reconsideración de su estructura desde el análisis del discurso. In L. BARBARA, Leyla y MOYANO, Estela Inés (Eds.). Textos y lenguaje académico. Exploraciones sistémico-funcionales en portugués y español. Los Polvorines: UNGS-PUCSP, 2011. 240 p. p.169-200.

MOYANO, Estela Inés. Nuevo conocimiento y construcción del autor. Tesis doctoral, Buenos Aires: Universidad de Buenos Aires, 2015a.

MOYANO, Estela Inés. Patrones de Realización de la Proyección en la Discusión de Artículos de Investigación Producidos en Español en Dos Disciplinas. Revista D.E.L.T.A., v 31, n 1, p.143-183, 2015b.

MOYANO, Estela Inés. Diseño e implementación de programas de lectura y escritura en el nivel universitario: principios y estrategias. Lenguas Modernas, n 50, p.47-72, 2017.

NAVARRO, Federico; SIMÕES, Alex Caldas. Potencial de Estructura Genérica en tesis de ingeniería eléctrica: Contrastes entre lenguas y niveles educativos. Revista Signos, n 51(100), p.306-329, 2019.

ROSE, David. Languages of Schooling: Embedding Literacy Learning with Genre-based Pedagogy. European Journal of Applied Linguistics, v 6, n 1, p. 5990, 2018a.

ROSE, David. Pedagogic register analysis: mapping choices in teaching and learning. Functional Linguistics, v 5, n 3, p. 1-33, $2018 \mathrm{~b}$.

ROSE, David. Building a pedagogic metalanguage I: curriculum genres. In MARTIN, J.R.; MATON, Karl; DORAN, Y.J. (Eds.). Accessing Academic Discourse: Systemic Functional Linguistics and Legitimation Code Theory, London: Routledge. 308 p, p.236-267, 2020a.

ROSE, David. Building a pedagogic metalanguage II: knowledge genres. In MARTIN, J.R.; MATON, Karl; DORAN, Y.J. (Eds.). Accessing Academic Discourse: Systemic Functional Linguistics and Legitimation Code Theory, London: Routledge. 308 p, p. 268-302, 2020b.

ROSE, David; MARTIN, J.R. Learning to Write, Reading to Learn. Genre, Knowledge and Pedagogy in the Sydney School. London: Equinox, 2012.

ROTHERY, seppoan. lisepis English acyisepiand

Learning) agedisepis Schools

VIDAL LIZAMA, Margarita. Aproximación al ensayo académico como género de formación en Ciencias Sociales: el caso de Sociología. D.E.L.T.A., v 36, n 4, p.1-26, 2020 .

VIGOTSKY, Leon. Pensamiento y lenguaje. Buenos Aires: Fausto, 1998.

WHITTAKER, Rachel; GARCÍA PAREJO, Isabel. Teacher Learning for European Literacy Education (TeL4ELE): genre-based pedagogy in five European countries. European Journal of Applied Linguistics, v 6, n 1, p.31-57, 2018.

\section{COMO CITAR ESSE ARTIGO}

VIDAL LIZAMA, Margarita. Aplicaciones del Programa Leer para Aprender en el nivel universitario: principios, desafíos y adaptaciones. Signo, Santa Cruz do Sul, v. 46, n. 86, maio 2021. ISSN 1982-2014. Disponível em: $<$ https://online.unisc.br/seer/index.php/signo/article/view/ 16031>.doi:https://doi.org/10.17058/signo.v46i86.16031. 